Series A

\author{
I. MATHEMATICA
}

383

\title{
FUNKTIONENRÄUME \\ IN DER KATEGORIE DER LIMESRÄUME
}

$$
\text { VON }
$$

E. BINZ und H. H. KELLER

Herrn Professor Dr. Rolf Nevanlinna zum 70. Geburtstag gewidmet 
Am 8. Oktober 1965 vorgelegt von P. J. Mrrberg und Olli Lehto 


\section{Funktionenräume in der Kategorie der Limesräume *}

\section{Einleitung}

Im Mittelpunkt der vorliegenden Arbeit steht die Menge ' $(X, Y)$ der stetigen Abbildungen eines Limesraumes $X$ in einen Limesraum $Y$. Durch Einführung einer Limesstruktur auf $\mathcal{C}(X, Y)$ wird dieser Funktionenraum selbst zu einem Limesraum gemacht. Es werden zwei Limitierungen auf $\mathcal{C}(X, Y)$ betrachtet, die beide durch die Struktur von ' $\mathcal{C}^{\prime}(X, Y)$ in natürlicher Weise bestimmt sind: die Limitierung $A_{c}$ der stetigen Konvergenz im ersten Abschnitt und die Limitierung $\Lambda_{s}$ der einfachen Konvergenz im dritten Abschnitt. Es handelt sich bei $\Lambda_{c}$ (bzw. $\Lambda_{s}$ ) um die gröbste Limitierung auf $\mathcal{C}(X, Y)$, für welche die kanonische Abbildung

$$
\omega: \mathcal{C}(X, Y) \times X \rightarrow Y
$$

stetig (bzw. getrennt stetig) ist. Sowohl $\Lambda_{c}$ als auch $\Lambda_{s}$ werden charakterisiert durch je eine universelle Eigenschaft in der Kategorie der Limesräume (Sätze 2 und 24). Die völlige formale Analogie der Aussagen dieser Sätze wird erreicht, indem auf der Produktmenge zweier Limesräume der gewöhnlichen Produktlimitierung eine feinere zur Seite gestellt wird, die wir die Limitierung der getrennten Stetigkeit nennen (Satz 22).

Der zweite Abschnitt behandelt den Fall, wo in $Y$ stetige algebraische Operationen definiert sind, die sich dann auf den Limesraum

$$
\left(\mathcal{C}(X, Y), A_{c}\right)
$$

übertragen. In diesem Zusammenhang werden zur Abkürzung der siprechweise die Begriffe Limesmonoid, Limesgruppe, Limesring etc. eingeführt.

Für die Limitierung der stetigen Konvergenz sei insbesondere auf die Arbeit von C. H. Cook und H. R. Fischer [3] verwiesen. Unter dem Namen "pseudotopologie de la convergence locale» wird diese Limesstruktur von Andrée Bastiani in [1] benutzt, desgleichen von E. Binz in [2]. Ferner wird sie von H. H. Keller in [5] kurz betrachtet.

* Mit Dankbarkeit sei erwähnt, dass diese Arbeit im Rahmen einer von der Fritz Hoffman - La Roche-Stiftung zur Förderung wissenschaftlicher Arbeitsgemeinschaften in der Schweiz unterstützten Forschungsgruppe entstanden ist. 
Es sei noch darauf aufmerksam gemacht, dass $\Lambda_{c}$, im Gegensatz zu $A_{s}$, im allgemeinen im Rahmen der topologischen Räume nicht konstruiert werden kann; m. a.W. selbst wenn $X$ und $Y$ topologische Räume sind, ist $\Lambda_{c}$ im allgemeinen keine Topologie auf $\ell(X, Y)$. Eine entsprechende Bemerkung gilt übrigens auch für die erwähnte Limitierung der getrennten Stetigkeit. Für die in dieser Arbeit durchgeführten Konstruktionen ist es somit wesentlich, dass in der Kategorie der von H. R. Fischer [4] eingeführten Limesräume gearbeitet wird. Da Limesräume nicht als allgemein bekannt vorausgesetzt werden können, schicken wir eine kurze Zusammenfassung der später gebrauchten Begriffe und Bezeichnungen voraus.

0.1. Sei $X$ eine nicht-leere Menge. Eine Limesstruktur oder Limitierung $A$ auf $X$ ordnet jedem Punkt $x \in X$ eine Menge $\Lambda(x)$ von Filtern in $X$ so zu, dass die folgenden Axiome erfüllt sind:

$(\operatorname{Lim} 1)$

$$
\begin{gathered}
\Phi \in \Lambda(x), \quad \Phi \leq \Psi \Rightarrow \Psi \in \Lambda(x) \\
\Phi \in \Lambda(x), \Psi \in \Lambda(x) \Rightarrow \Phi \wedge \Psi \in A(x) \\
\text { Für jedes } x \in X \text { ist } \dot{x} \in \Lambda(x) .
\end{gathered}
$$

Dabei bedeutet $\Phi \leq \Psi$, dass $\Psi$ ein Filter in $X$ ist, welcher feiner als $\Phi$ ist: $\Phi \wedge \Psi$ bezeichnet unter den Filtern in $X$, die gröber als $\Phi$ und $\Psi$ sind, den feinsten; mit $\dot{x}$ ist der durch $x$ bestimmte triviale Ultrafilter in $X$ bezeichnet. Eine Menge $A(x)$ von Filtern, die den Axiomen (Lim 1) und (Lim 2) genügt, heisst ein Filterideal.

Die Limitierung $A$ auf $X$ heisst separiert, wenn ausserdem gilt

$$
\text { Für } x \neq y \text { ist } \Lambda(x) \cap \Lambda(y) \text { leer. }
$$

Das Paar $(X, \Lambda)$ heisst ein Limesraum. Statt $\Phi \in \Lambda(x)$ schreiben wir im folgenden stets $\Phi \rightarrow x \in(X, \Lambda)$ oder $\Phi \rightarrow x \in X$, falls $X$ selbst schon den Limesraum bezeichnet und für die Limitierung kein separates Symbol verwendet wird.

0.2. Seien $X$ und $Y$ Limesräume. Eine Abbildung $f: X \rightarrow Y$ heisst stetig, wenn für jedes $x \in X$ und jeden Filter $\Phi$ in $X$ gilt

$$
\Phi \rightarrow x \in X \Rightarrow f(\Phi) \rightarrow f(x) \in Y .
$$

Dabei bezeichnet $f(\Phi)$ den Bildfilter von $\Phi$ vermöge $f$.

Es ist klar, dass für jeden Limesraum $X$ die identische Abbildung $\operatorname{id}_{X}: X \rightarrow X$ stetig ist. Ebenso ist für drei Limesräume $X, Y$ und $Z$ die Komposition $g \circ f: X \rightarrow Z$ von zwei stetigen Abbildungen $f: X \rightarrow Y$ und $g: Y \rightarrow Z$ stetig. Daraus folgt, dass die Limesräume die Objekte 
einer Kategorie ' $C$ sind, wenn die stetigen Abbildungen zwischen Limesräumen als die Morphismen von $\mathcal{C}$ erklärt werden. Wir nennen $\mathcal{C}$ kurz die Kategorie der Limesräume. Für je zwei Objekte $X, Y$ von $\bigodot$ wird die Menge der Morphismen von $X$ in $Y$ allgemein mit ${ }^{\top}(X, Y)$ bezeichnet. Die Iscmorphismen in der Kategorie ${ }$ werden auch Homöomorphismen genannt.

Jeder topologische Raum $X$ trägt in natürlicher Weise eine Limitierung 1. indem für jedes $x \in X$ das Filterideal $A(x)$ aus den für die Topologie von $X$ gegen $x$ konvergenten Filtern besteht. Die Kategorie der topolcgischen Rälme ist in diesem Sinne als Unterkategorie von $C$ aufzufassen.

0.3. Sei $X$ eine nicht-leere Menge. Eine Limitierung $\Lambda^{\prime}$ heisst feiner als eine Limitierung $\Lambda$ auf $X$, (oder auch $\Lambda$ gröber als $\Lambda^{\prime}$ ), wenn

$$
\mathrm{id}_{X}:\left(X, A^{\prime}\right) \rightarrow(X, A)
$$

stetig ist. Dies ist genau dann der Fall, wenn man $A^{\prime}(x) \subset A(x)$ für jedes $x \in X$ hat.

Nun seien $X$ eine nicht-leere Menge und $\left(Y_{\imath}, A_{\imath}\right)_{\iota \in J}$ eine Familie von Limesräumen. Wir betrachten die folgenden beiden Situationen:

1) Für jedes $\iota \in J$ sei eine Abbildung $f_{\imath}: X \rightarrow Y$, gegeben. Dann gibt es unter allen Limitierungen $A$ auf $X$, für welche alle Abbildungen

$$
f_{\imath}:(X, A) \rightarrow\left(Y_{\imath}, A_{\imath}\right), \quad \iota \in J,
$$

stetig sind, eine gröbste, $\Lambda_{0}$. Sie heisse die von der Familie $\left(f_{\iota}\right)_{1 \in J}$ induzierte Limitierung. Ist $S$ ein beliebiger Limesraum, so ist eine Abbildung $u: S \rightarrow\left(X, A_{0}\right)$ genau dann stetig, wenn $f_{\iota} \circ u: S \rightarrow\left(Y, A_{\imath}\right)$ für jedes $\imath \in J$ stetig ist.

2) Für jedes $\iota \in J$ sei eine Abbildung $g, Y, \rightarrow X$ gegeben. Dann existiert unter allen Limitierungen $A$ auf $X$, für welche alle Abbildungen

$$
g_{\imath}:\left(Y, \Lambda_{\imath}\right) \rightarrow(X, \Lambda), \quad \iota \in J,
$$

stetig sind, eine feinste, $\Lambda^{0}$, die von der Familie $\left(g_{1}\right)_{1 \in J}$ erzeugte Limitierung. Für jedes $x \in X$ wird das Filterideal $\Lambda^{0}(x)$ erzeugt durch den Ultrafilter $\dot{x}$ und alle Filter der Form $g_{\imath}\left(\Psi_{\imath}\right), \Psi_{\imath} \rightarrow y_{\imath} \in\left(Y_{\imath}, A_{\imath}\right)$. Dabei durchläuft $\iota$ die Indexmenge $J$ und für jedes $\iota \in J$ durchläuft $y$, die Urbildmenge $g_{\imath}^{-1}(x) \subset Y_{\imath}$. Ist $T$ irgend ein Limesraum, so ist eine Abbildung $v:\left(X, A^{0}\right) \rightarrow T$ genau dann stetig, wenn $v \circ g_{\imath}:\left(Y_{,}, A_{\imath}\right) \rightarrow T$ für jedes $\iota \in J$ stetig ist.

0.4. Seien $(X, A)$ ein Limesraum und $A$ eine nicht-leere Teilmenge von $X$. Die durch die Inklusion $i: A \rightarrow X$ auf $A$ induzierte Limitierung (auch die von $A$ auf $A$ induzierte genannt), werde der Einfachheit halber 
auch mit $\Lambda$ bezeichnet. Dann heisst $(A, \Lambda)$ Unterraum des Limesraumes $(X, \Lambda)$.

Ist $\left(X_{\imath}, \Lambda_{\iota}\right)_{\iota} \in J$ eine Familie von Limesräumen, so wird auf dem cartesischen Produkt $\prod_{\iota \in J} X_{\iota}$ die Produktlimitierung $\prod_{\iota \in J} \Lambda_{\iota}$ definiert als die von der Familie $\left(p_{\iota}\right)_{, \in J}$ der Projektionen $p_{\varkappa}: \prod_{\iota \in J} X_{\iota} \rightarrow X_{\iota}$ induzierte Limitierung, und $\prod_{\iota \in J}\left(X_{\iota}, \Lambda_{\iota}\right)=\left(\prod_{\iota \in J} X_{\iota}, \prod_{\iota \in J} \Lambda_{\iota}\right)$ heisst das Produkt der Familie $\left(X_{\iota}, A_{\iota}\right)_{\iota} \in J$ von Limesräumen.

0.5. Folgende Bezeichnungen werden ganz generell verwendet.

Sind $X$ und $Y$ nicht-leere Mengen, so ist $\mathcal{F}(X, Y)$ die Menge aller Abbildungen von $X$ in $Y$. Diese wird mit der Produktmenge $I^{X}$ identifiziert.

Ist $H$ eine nicht-leere Teilmenge von $\mathcal{F}(X, Y)$, so bezeichnet allgemein das Symbol $\omega$ die natürliche Abbildung

$$
\omega: H \times X \rightarrow Y,
$$

definiert durch $\omega(u, x)=u(x)$ für jedes $u \in H$ und jedes $x \in X$. Falls ferner eine Menge $M$ und eine Abbildung $f: M \rightarrow H$ gegeben sind, so heisse

$$
\tilde{f}=\omega \circ\left(f \times \operatorname{id}_{X}\right): M \times X \rightarrow Y
$$

die zu $f$ assoziierte Abbildung. Es ist $\tilde{f}(p, x)=f(p)(x)$ für jed€s $p \in M$ und jedes $x \in X$.

\section{Die Limitierung der stetigen Konvergenz}

1.1. Satz 1. Seien $X$ und $Y$ Limesräume. Unter allen Limitierungen $\Lambda$ auf $\mathcal{C}(X, Y)$ für welche die natürliche Abbildung

$$
\omega:(\mathscr{C}(X, Y), A) \times X \rightarrow Y
$$

stetig ist, gibt es eine gröbste, $\Lambda_{c}$. Diese ist genau dann separiert, wenn $Y^{-}$ separiert ist.

Man definiere $\Lambda_{c}$ durch die Forderung, dass für jedes $u \in \mathscr{C}(X, Y)$ und jeden Filter $\Theta$ auf $\mathcal{C}(X, Y)$ die Konvergenz $\Theta \rightarrow u \in\left(\mathcal{C}(X, Y), A_{c}\right)$ genau dann bestehe, wenn für jedes $x \in X$ und jeden Filter $\Phi$ auf $X$ gilt:

$$
\Phi \rightarrow x \in X \Rightarrow \omega(\Theta \times \Phi) \rightarrow u(x) \in Y .
$$

Man verifiziert, dass $\Lambda_{c}$ eine Limitierung auf $\mathcal{C}(X, Y)$ ist, und dass diese die im ersten Teil von Satz 1 geforderte Eigenschaft besitzt. Der zweite Teil von Satz 1 ergibt sich unmittelbar aus der Konstruktion von $\Lambda_{c}$. Für die Einzelheiten sei auf [3] verwiesen. 
In Übereinstimmung mit [3] nennen wir $\Lambda_{c}$ die Limitierung der stetigen Konvergenz auf $\varphi_{(X, Y)}$ und schreiben fortan ${ }^{C}(X, Y)$ statt $\left.(X, Y), A_{c}\right)$.

1.2. Es seien $H$ eine nicht-leere Teilmenge von ' $(X, Y)$ und

$$
i: H \rightarrow{ }^{\prime}(X, Y)
$$

die Inklusionsabbildung. Wir setzen $\left(H, A_{c}\right)=H_{c}$. Die Limesstruktur 1. besitzt die folgende universelle Eigenschaft:

Satz 2. Es seien $X$ und $Y$ Limesräume und $H_{c}$ ein Unterraum von ' ${ }_{c}(X, Y)$. Für jeden Limesraum $S$ ist eine Abbildung $f: S \rightarrow H_{c}$ dann und nur dann stetig, wenn die assoziierte Abbildung $\tilde{f}: S \times X \rightarrow Y$ stetig ist.

Wenn $f$ stetig ist, so ist $\tilde{f}=\omega \circ\left(i \circ f \times \mathrm{id}_{X}\right)$ stetig, weil $i$ und $\omega$ stetig sind. Ist umgekehrt $\tilde{f}$ stetig, so seien $s \in S$ und $x \in X$ beliebige Punkte, ferner $\Sigma, \Phi$ beliebige Filter auf $S$ bzw. $X$, für die $\Sigma \rightarrow s \in S$, $\Phi \rightarrow x \in X$. Nach Voraussetzung über $\tilde{f}$ hat man

$$
\omega(i(f(\Sigma)) \times \Phi)=\tilde{f}(\Sigma \times \Phi) \rightarrow \tilde{f}(s, x)=f(s)(x) \in Y .
$$

Aus der Konstruktion von $A_{c}$ folgt $i(f(\Sigma)) \rightarrow i(f(s)) \in \mathcal{C}_{c}(X, Y)$. Daher ist $i \circ f: S \rightarrow{ }^{\prime}(X, Y)$ stetig, und somit auch $f: S \rightarrow H_{c}$. Damit ist Satz 2 bewiesen.

1.3. Tatsächlich ist die Limitierung $A_{c}$ auf jeder nicht-leeren Teilmenge von ' $C(X, Y)$ durch die in Satz 2 ausgedrückte universelle Eigenschaft charakterisiert.

Satz 3. Es seien $X, Y$ Limesräume, $H$ eine nicht-leere Teilmenge von $'(X, Y)$ und $A$ eine Limitierung auf $H$ mit der Eigenschaft: Für jeden Limesraum $S$ ist eine Abbildung $f: S \rightarrow(H, A)$ dann und nur dann stetig, uenn $\tilde{f}: S \times X \rightarrow Y$ stetig ist. Unter diesen Voraussetzungen ist 1 mit $\Lambda_{c}$ identisch.

Falls $A$ der Voraussetzung von Satz 3 genügt, so ist mit id $\operatorname{id}_{H}:(H, A)$ $\rightarrow(H, A)$ auch $\widetilde{\mathrm{id}}_{H}:(H, A) \times X \rightarrow Y$ stetig, und daher nach Satz 2 auch $\operatorname{id}_{H}:(H, A) \rightarrow H_{c}$. Umgekehrt folgt aus der Stetigkeit von $\widetilde{\mathrm{id}}_{H}=$ (1) $: H_{c} \times X \rightarrow Y$, nach Voraussetzung über $\Lambda$, dass $\operatorname{id}_{H}: H_{c} \rightarrow(H, \Lambda)$ stetig ist.

Korollar. Es seien $U, X, Y$ Limesräume und $H$ eine Teilmenge von "(X,Y). Eine bijektive Abbildung $u: U \rightarrow H_{c}$ ist dann und nur dann ein Homöomorphismus, wenn die folgenden beiden Bedingungen erfüllt sind:

(i) $\tilde{u}: U \times X \rightarrow Y$ ist stetig.

(ii) Für jeden Limesraum $S$ und jede Abbildung $f: S \rightarrow U$ impliziert die Stetigkeit von $\widetilde{u \circ f}: S \times X \rightarrow Y$ diejenige von $f$. 
Aufgrund von Satz 3 ist $u$ genau dann ein Homöomorphismus, wenn für jeden Limesraum $S$ und jede Abbildung $f: S \rightarrow U$ gilt:

$$
\widetilde{u \circ f} \text { stetig } \Leftrightarrow f \text { stetig. }
$$

Mit dieser Bedingung äquivalent ist aber das Paar (i), (ii).

1.4. Es bezeichne $K$ die Menge der konstanten Abbildungen von $X$ in $Y$. Die natürliche Bijektion $\varphi: Y \rightarrow K$ ist definiert durch $\varphi(y)(x)=y$ für jedes $y \in Y$ und jedes $x \in X$.

Satz 4. Es seien $X, Y$ Limesräume und $K$ die Menge der konstanten Abbildungen von $X$ in $Y$. Die natürliche Bijektion $\varphi: Y \rightarrow K_{c}$ ist ein Hcmöcmorphismus.

Die zu $\varphi$ assoziierte Abbildung $\tilde{\varphi}: Y \times X \rightarrow Y$ ist die Projektion. Diese ist stetig, und daher auch $\varphi$. Andrerseits sind $\omega: K_{c} \times X \rightarrow Y$ und die Projektion $K_{c} \times X \rightarrow X$ stetig, folglich auch

$$
\varphi^{-1} \times \mathrm{id}_{X}: K_{c} \times X \rightarrow Y \times X \text {. }
$$

Hieraus folgt die Stetigkeit von $\varphi^{-1}: K_{c} \rightarrow Y$.

1.5. Seien $X, Y$ und $Z$ Limesräume. Nach Satz 2 ist eine Abbildung $f: X \rightarrow \mathcal{C}_{c}(Y, Z)$ genau dann stetig, wenn $\alpha(f)=\tilde{f}: X \times Y \rightarrow Z$ stetig ist. Folglich ist $\alpha$ eine Bijektion von $C\left(X, C_{c}(Y, Z)\right)$ auf $\mathcal{C}(X \times Y, Z)$.

Satz 5. Für drei Limesräume $X, Y$ und $Z$ ist die durch $\alpha(f)=\tilde{f}$ definierte Abbildung $\alpha: C_{c}\left(X, C_{c}(Y, Z)\right) \rightarrow{ }^{C} C_{c}(X \times Y, Z)$ ein Homöomorphismus.

Der Beweis ergibt sich durch wiederholte Anwendung von Satz 2 wie folgt. Zunächst zieht die Stetigkeit der natürlichen Abbildungen

$$
\mathcal{C}_{c}\left(X, \mathcal{C}_{c}(Y, Z)\right) \times X \times Y \stackrel{\omega \times \mathrm{id}_{Y}}{\longrightarrow} \mathcal{C}_{c}(Y, Z) \times Y \stackrel{\left({ }^{\prime \prime}\right.}{\longrightarrow} Z
$$

die Stetigkeit von $\alpha$ nach sich. Andrerseits ist

$$
\text { (): } \mathscr{C}_{c}(X \times Y, Z) \times X \times Y \rightarrow Z
$$

stetig: und daher auch

$$
\widetilde{a^{-1}}:{ }^{c}(X \times Y, Z) \times X \rightarrow{ }_{c}(Y, Z) .
$$

Somit ist auch $\alpha^{-1}$ stetig.

1.6. Satz 6. Für drei Limesräume $X, Y, Z$ ist die durch die Komposition $(v, u) \longrightarrow v \circ u$ definierte natürliche Abbildung von $\mathcal{C}_{c}(Y, Z) \times{ }^{{ } C_{c}}(X, Y)$ in $\Upsilon_{c}(X, Z)$ stetig. 
Wiederum nach Satz 2 hat man zu zeigen, dass die Abbildung $(v, u, x) \longrightarrow(v \circ u)(x)=v(u(x))$ von $\ell_{c}(Y, Z) \times{ }^{c}(X, Y) \times X$ auf $Z$ stetig ist. Dies ergibt sich durch die Zerlegung in die stetigen Abbildungen

$$
e_{c}(Y, Z) \times \bigodot_{c}(X, Y) \times X \rightarrow \bigodot_{c}(Y, Z) \times Y \rightarrow Z,
$$

definiert durch

$$
(v, u, x) \longrightarrow(v, u(x)) \longrightarrow v(u(x))
$$

1.7. Satz 7. Seien $X$ ein Limesraum, $\left(Y_{,}\right)_{1} \in J$ eine Familie von Limesräumen und $q_{\varkappa}: \prod_{\iota} \in Y_{\iota} \rightarrow Y_{*}$ die Projektionen. Die durch die Zuordnung $u \longrightarrow(q, u)_{\iota} \in J$ definierte natürliche Bijektion

$$
\beta: e_{c}\left(X, \prod_{\iota \in J} Y_{\imath}\right) \rightarrow \prod_{\iota \in J} \ell_{c}\left(X, Y_{\imath}\right)
$$

ist ein Homöomorphismus.

Seien

$$
p_{\varkappa}: \prod_{\iota \in J} \circlearrowright\left(X, Y_{\iota}\right) \rightarrow \bigodot\left(X, Y_{\varkappa}\right), \quad x \in J
$$

die Projektionen. Durch Anwendung von Satz 2 ergibt sich zunächst, dass für jedes $x \in J$ die Abbildungen

$$
e_{c}\left(X, \prod_{1 \in J} Y_{\imath}\right) \times X \stackrel{\omega}{\rightarrow} \prod_{\imath \in J} Y, \stackrel{q_{\varkappa}}{\rightarrow} Y_{*}
$$

stetig sind, und hieraus, dass

$$
p_{\varkappa} \circ \beta:{ }^{C_{c}}\left(X, \prod_{\iota \in J} Y_{\iota}\right) \rightarrow{ } e_{c}\left(X, Y_{\varkappa}\right), \approx \in J,
$$

stetig ist, und damit $\beta$ selbst.

Andrerseits ist das folgende Diagramm natürlicher Abbildungen für jedes $x \in J$ kommutativ.

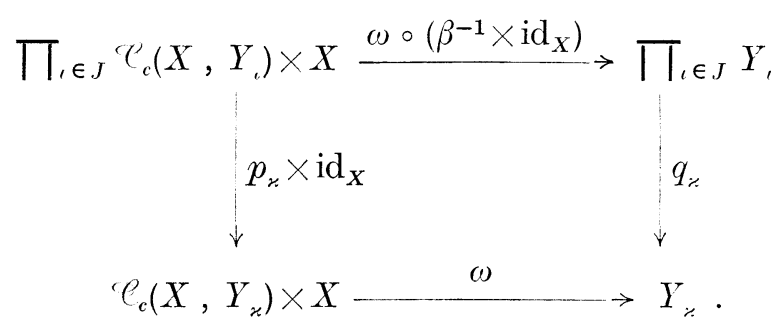

Die Stetigkeit von $\omega \circ\left(\beta^{-1} \times \mathrm{id}_{X}\right)$ folgt nach Definition der Produktlimitierung auf $\prod_{\imath \in J} Y_{\imath}$ aus der Stetigkeit der übrigen Abbildungen. Nach Satz 2 ist $\beta^{-1}$ stetig. 
1.8. Das Produkt einer Familie $\left(f_{1}\right)_{t} \in J$ von Abbildungen $f_{1}: X, \rightarrow Y$, zwischen Limesräumen,

$$
\prod_{, \in J} f,: \prod_{, \in J} X_{,} \rightarrow \prod_{, \in J} Y_{,}
$$

definiert durch

$$
\left(\prod_{1} \in J, f,\left(x_{1}\right)_{1 \in J}=(f,(x,)), \in J,\right.
$$

ist genau dann stetig, wenn $f$, für jedes $\imath \in J$ stetig ist.

Satz 8. Seien $\left(X_{1}\right)_{1 \in J}$ und $\left(Y_{1}\right)_{1} \in J$ wwei Familien von Limesräumen mit der gleichen Indexmenge $J$. Die durch $\left(f_{i}\right)_{, \in J} \rightarrow \prod_{, \in J} f$ definiente naturliche Injeltion

$$
\gamma: \prod_{, \in J} \varphi_{c}\left(X_{1}, Y_{,}\right) \rightarrow \iota_{c}\left(\prod_{, \in J} X_{1}, \prod_{, \in J} Y_{,}\right)
$$

bildet den Raum $\prod_{, \in J} C_{c}\left(X_{1}, Y_{1}\right)$ homöomorph auf einen Lnterraum ion $\varphi_{c}\left(\prod_{, \in J} X_{,}, \prod_{, \in J} Y_{2}\right) a b$.

As Produkt der stetigen Abbildungen

$$
\omega: \Upsilon_{c}\left(X_{1}, Y_{1}\right) \times X_{1} \rightarrow Y_{1} \quad(\iota \in J)
$$

ist

$$
\tilde{\gamma}: \prod_{1 \in J}{ }^{\prime}{ }_{c}\left(X_{,}, Y_{1}\right) \times \prod_{, \in J} X_{,} \rightarrow \prod_{, \in J} Y_{,}
$$

stetig und daher auch $\gamma$.

Nach dem Korollar von Satz 3 hat man noch zu zeigen, dass für jeden Limesraum $S$ eine Abbildung

$$
g=\left(g_{1}\right)_{1 \in J}: S \rightarrow \prod_{1 \in J}{ }^{\prime} C_{c}\left(X_{,}, Y_{1}\right)
$$

stetig ist, wenn nur

$$
\widetilde{\gamma \circ g}: S \times \prod_{, \in J} X, \rightarrow \prod_{, \in J} Y,
$$

stetig ist. Sei also vorausgesetzt, dass

$$
\widetilde{\gamma \circ g}:\left(s,\left(x_{1}\right)_{1} \in J\right) \longrightarrow\left(g_{1}(s)\left(x_{1}\right)\right)_{1} \in J
$$

stetig sei. Dann ist für jedes $\iota \in J$ die Abbildung

$$
\tilde{g}_{1}: S \times X_{1} \rightarrow Y_{,} \quad\left(\tilde{g}_{1}\left(s, x_{1}\right)=g_{1}(s)\left(x_{1}\right)\right),
$$

stetig, und daher nach Satz 2 auch

$$
g_{\ell}: S \rightarrow \Upsilon_{c}\left(X_{1}, Y_{1}\right)
$$

was äquivalent zur Stetigkeit von $g$ ist.

1.9. Es bezeichne $C$ die Kategorie, deren Objekte die Limesräume und deren Morphiءmen die stetigen Abbildungen zwischen Limesräumen 
sind. Sind $X, Y$ Limesräume, so ist die Morphismenmenge $\mathscr{C}(X, Y)$, rersehen mit der Limitierung $A_{c}$ der stetigen Konvergenz, selbst wieder ein Objekt $\mho_{c}(X, Y)$ der Kategorie $\mathcal{~}$.

Jeder feste Limesraum $S$ bestimmt in natürlicher Weise einen kovarianten Funktor ' $C_{S}$ der Kategorie $C$ in sich. Für jeden Limesraum $X$ setze man nämlich ${ }^{C}(X)=\bigodot_{c}(S, X)$, und jedem Morphismus $f \in{ }^{\top}(X, Y)$ ordne man die Abbildung

$$
C_{S}(f): \varphi_{c}(S, X) \rightarrow \bigodot_{c}(S, Y)
$$

zu, die durch

$$
\bigodot_{S}(f)(u)=f \circ u \quad\left(u \in C^{\prime}(S, X)\right),
$$

definiert ist. Da

$$
\widetilde{\bigodot_{S}(f)}: \Upsilon_{c}(S, X) \times S \rightarrow Y
$$

stetig ist, so ist nach Satz 2 auch ' ${ }_{S}(f)$ stetig, also ein Morphismus:

$$
{ }^{\prime}{ }_{s}(f) \in C\left(C_{S}(X),{ }^{\prime} C_{S}(Y)\right) \text {. }
$$

Wan rerifiziert leicht, dass ' $C_{S}$ in der Tat die Eigenschaften eines kovarianten Funktors aufweist.

Fiir jedes Paar $X, Y$ von Limesräumen kann die Zuordnung $f^{\prime} \rightarrow(f)$ als eine Abbildung

$$
\Upsilon_{S}: C_{c}(X, Y) \rightarrow{ }^{\prime}{ }_{c}\left({ }{ }_{S}(X),{ }^{C}{ }_{S}(Y)\right)
$$

aufgefasst werden. Diese ist injektiv, wie man sofort einsieht, und stetig nach Satz 2, weil die natürliche Abbildung

$$
\widetilde{\Upsilon}_{S}:{ }^{\prime}(X, Y) \times \Upsilon_{c}(S, X) \rightarrow \Upsilon_{c}(S, Y),
$$

die durch die Komposition $(f, u) \longrightarrow f \circ u$ gegeben ist, nach Satz 6 stetig ist. Es gilt jedoch sogar der

Satz 9. Es seien $S, X, Y$ Limesräume. Der kovariante Funktor ' $\mathcal{C}_{S}$ in der Kategorie ' $\mathcal{C}$ der Limesräume bildet den Raum $\mathcal{C}_{c}(X, Y)$ homöomorph auf einen Unterraum von $\mathcal{C}_{c}\left(C_{S}(X),{ }^{C} C_{S}(Y)\right)$ ab.

Nach dem Korollar von Satz 3 hat man noch zu zeigen, dass für jeden Limesraum $T$ eine Abbildung $g: T \rightarrow{ }^{C}(X, Y)$ stetig ist, falls

$$
\widetilde{C_{S} \circ g}: T \times{ }^{\prime}(S, X) \rightarrow{ }^{\prime}(S, Y) \text {, }
$$

definiert durch $(t, u) \rightarrow g(t) \circ u$, stetig ist. Wir setzen also voraus, dass diese letzte Abbildung stetig sei. Nun seien $K_{c} \subset{ }^{c}(S, X), K_{c}^{\prime} \subset \ell_{c}(S, Y)$ die Unterräume, bestehend aus den konstanten Funktionen, und 
$\varphi: X \rightarrow K_{c}, \psi: Y \rightarrow K_{c}^{\prime}$ die kanonischen Homöomorphismen (Satz 4). Wir betrachten die Sequenz stetiger Abbildungen:

$$
T \times X \stackrel{\mathrm{id}_{T} \times \varphi}{\rightarrow} T \times K_{c} \rightarrow K_{c}^{\prime} \stackrel{\psi^{-1}}{\rightarrow} Y
$$

wobei die mittlere die Restriktion von $\overline{\bigodot_{S} \circ g}$ auf $T \times K_{c}$ ist (mit Werten in $K_{c}^{\prime}$ ). Man verifiziert, dass die Komposition dieser Abbildungen gerade $\tilde{g}:(t, x) \longrightarrow g(t)(x)$ ist. Somit ist nach Satz 2 auch $g$ stetig.

Die Aussage des Satzes 5, welche für die Limitierung der stetigen Konvergenz charakteristisch ist, lässt sich mit Hilfe des kovarianten Funktors $C_{S}$ wie folgt ausdrücken:

Satz 10. Seien $S$ und $T$ zwei feste Limesräume. Wird für jeden Limesraum $X$ vermöge des natürlichen Homöomorphismus $\alpha$ der Raum $\ell_{c}\left(S, \ell_{c}(T, X)\right)$ mit $\ell_{c}(S \times T, X)$ identifiziert, so sind die Funktoren $e_{\mathrm{S}} \circ \mathscr{C}_{\mathrm{T}}$ und $e_{\mathrm{S} \times \mathrm{T}}$ identisch:

$$
e_{S} \circ e_{T}={ }^{\prime} e_{S \times T} .
$$

Jedem festen Limesraum $S$ entspricht auch ein kontravarianter Funktor $e^{S}$ von $C$ in sich, indem man setzt ${ }^{C}(X)=\ell_{c}(X, S)$, und jedem Morphismus $f \in \mathcal{C}(X, Y)$ den Morphismus $e^{S}(f) \in \mathcal{C}^{\prime}\left(\mathcal{C C S}^{\mathrm{S}}(Y), \mathcal{C}^{\mathrm{S}}(X)\right)$ zuordnet, der definiert ist durch

$$
{ }^{\mathcal{C}}(f): v \longrightarrow v \circ f \quad\left(v \in \mathcal{C}^{\mathscr{C}}(Y, S)\right) .
$$

Dass für jedes Paar $X, Y$ von Limesräumen die Abbildung

$$
e^{s}: e_{c}(X, Y) \rightarrow e_{c}\left(e^{s}(Y), e^{s}(X)\right)
$$

stetig ist, folgt wie oben.

Satz 11. Es seien $S, X, Y$ Limesräume. Der kontravariante Funktor $e^{s}$ in der Kategorie $C$ der Limesräume bildet $C_{c}(X, Y)$ stetig in $e_{c}\left(e^{S}(Y), e^{s}(X)\right) a b$.

\section{Stetige algebraische Operationen}

2.1. Sei $F$ eine nicht-leere Menge. Eine innere Verknüpfung in $F$ ist eine Abbildung

$$
\mu: F \times F \rightarrow F
$$

Ist ausserdem eine weitere nicht-leere Menge $A$ gegeben, so heisse eine Abbildung

$$
\pi: A \times F \rightarrow F
$$


eine äussere Verknüpfung in $F$ mit dem Operatorenbereich $A$. Innere und äussere Verknüpfungen in $F$ werden im folgenden als algebraische Operationen in $F$ bezeichnet.

Ist $X$ eine beliebige nicht-leere Menge, so überträgt sich in natürlicher Weise jede in $F$ erklärte innere bzw. äussere Verknüpfung, $\mu$ bzw. $\pi$, auf die Menge $\mathcal{F}(X, F)=F^{X}$ aller Abbildungen von $X$ in $F$, indem man

$$
\mu_{*}: \mathcal{F}(X, F) \times \mathcal{F}(X, F) \rightarrow \mathcal{F}(X, F),
$$

bzw.

$$
\pi_{*}: \mathcal{F}(X, A) \times \mathcal{F}(X, F) \rightarrow \mathcal{F}(X, F),
$$

definiert durch

$$
\mu_{*}(u, v)(x)=\mu(u(x), v(x))
$$

bzw.

$$
\pi_{*}(\alpha, u)(x)=\pi(\alpha(x), u(x)),
$$

für beliebige Elemente $u, v \in \mathcal{F}(X, F), \alpha \in \mathcal{F}(X, A), x \in X$. Überdies induziert $\pi$ in $\mathcal{F}(X, F)$ eine äussere Verknüpfung

$$
\pi_{*}^{\prime}: A \times \mathcal{F}(X, F) \rightarrow \mathcal{F}(X, F),
$$

welche als Restriktion von $\pi_{*}$ auf $A \times \mathcal{F}(X, F)$ betrachtet werden kann. falls $A$ mit der Menge der konstanten Abbildungen von $X$ in $A$ identifiziert wird.

2.2. Nun seien die auftretenden Mengen $F, A, X$ Limesräume. Für jede stetige innere bzw. äussere Verknüpfung in $F, \mu$ bzw. $\pi$, bildet $\mu_{*}$ bzw. $\pi_{*}$ die Menge $\mathcal{C}(X, F) \times{ }^{\complement} \mathcal{C}(X, F)$, bzw. ' $C(X, A) \times{ }^{C}(X, F)$, in $\mathscr{C}(X, F)$ ab, so dass man, was im folgenden geschehen soll, die induzierte Verknüpfung $\mu_{*}$, bzw. $\pi_{*}$, als Abbildung

$$
\mu_{*}:{ }^{\prime} C_{c}(X, F) \times{ }^{\prime} C_{c}(X, F) \rightarrow{ }^{\prime} C_{c}(X, F),
$$

bzw.

$$
\pi_{*}:{ }^{\prime} C_{c}(X, A) \times{ }^{\prime} C_{c}(X, F) \rightarrow{ }^{\prime} \bigodot_{c}(X, F),
$$

betrachten kann.

Satz 12. Seien $X$ und $F$ Limesräume. Die von einer stetigen inneren, bzw. äusseren Verknüpfung, $\mu$ bzw. $\pi$, in $F$ induzierte Verknüpfung, $\mu_{*}$ bzw. $\pi_{*}$, in $\bigodot_{c}(X, F)$ ist stetig.

Nach Satz 7 ist nämlich die natürliche Abbildung

$$
\beta^{-1}: C_{c}(X, F) \times \bigodot_{c}(X, F) \rightarrow \bigodot_{c}(X, F \times F)
$$


ein Homöomorphismus; andrerseits ist die Abbildung $w \rightarrow \mu \circ w$ von $\bigodot_{c}(X, F \times F)$ in $\Upsilon_{c}(X, F)$ nach Satz 6 stetig, und daher auch $\mu_{*}$ als Komposition von beiden. Analog ergibt sich die entsprechende Aussage über $\tau_{*}$.

2.3. Falls $F$ eine algebraische Struktur trägt, deren Operationen assoziativen, kommutativen oder distributiven Gesetzen genügen, so sind die in.$(X, F)$ induzierten Operationen wieder assoziativ, kommutativ oder distributir. Dies gilt natürlich auch für die in $C_{c}(X, F)$ induzierten Operationen, wenn die zugrunde liegenden Räume Limesräume und die in $F$ definierten Operationen stetig sind.

Einen Limesraum $F$ mit stetiger, assoziativer innerer Verknüpfung wollen wir als Limesmonoid bezeichnen. Für ein Limesmonoid $F$ und einen Limestavm $X$ ist $\bigodot_{c}(X, F)$, mit der induzierten inneren Verknüpfung versehen, ein Limesmonoid. Falls $F$ ein Einselement $e$ besitzt, so ist $e_{*}=q(e) \in C_{c}(X, F)$ Einselement von $\varrho_{c}(X, F)$. Dabei ist

$$
\varphi: F \rightarrow C_{c}(X, F)
$$

die natürliche Einbettung, also $e_{*}(x)=e$ für jedes $x \in X$. Ein Element $u \in(X, F)$ ist genau dann invertierbar, wenn $u(x)$ für jedes $x \in X$ invertierbar in $F$ ist.

2.t. Unter einer Limesgruppe wollen wir einen Limesraum $F$ verstehen, der so mit einer Gruppenstruktur versehen ist, dass die Gruppenmultiplikation

und die Inversion

$$
\mu: F \times F \rightarrow F
$$

$$
i: F \rightarrow F \quad(\mu(i(y), y)=\mu(y, i(y))=e)
$$

stetig sind.

Satz 13. Seien $X$ ein Limesraum und $F$ eine Limesgruppe. Dann ist $C_{c}(X, F)$, versehen mit der induzierten inneren Verknüpfung, eine Limesgruppe.

Nach Satz 12 ist die Gruppenmultiplikation $\mu_{*}$ stetig. Es bleibt zu zeigen, dass dies auch für die Inversion

$$
i_{*}: \mathscr{C}_{c}(X, F) \rightarrow \mathscr{C}_{c}(X, F),
$$

definiert durch $i_{*}(u)(x)=i(u(x))$ für jedes $u \in \mathcal{C}_{c}(X, F)$ und jedes $x \in X$, zutrifft. Nun ist $\widetilde{i_{*}}$ die Komposition der Abbildungen

$$
\iota_{c}(X, F) \times X \stackrel{\omega}{\longrightarrow} F \stackrel{i}{\longrightarrow} F,
$$

also stetig. Nach Satz 2 ist $i_{*}$ stetig. 
2.5. Unter einem Limesring wollen wir einen Limesraum mit Ringstruktur verstehen, der für die Addition eine (abelsche) Limesgruppe und für die Multiplikation ein Limesmonoid ist. Satz 12 und Satz 13 liefern dann den

Satz 14. Seien $X$ ein Limesraum und $R$ ein Limesring. Dann ist ' ${ }_{c}(X, R)$, versehen mit den induzierten Ringoperationen, ein Limesring.

Als Limeskörper sei ein Limesraum mit Körperstruktur bezeichnet, der für die zugrunde liegende Ringstruktur ein Limesring ist, und für den die Inversion (definiert auf dem Unterraum der von $O$ verschiedenen Elemente) stetig ist.

Falls $X$ ein Limesraum und $K$ ein Limeskörper ist, so ist ' ${ }^{c}(X, K)$, mit den induzierten algebraischen Operationen versehen, zwar ein Limesring. im allgemeinen jedoch kein Limeskörper.

2.6. Ein Limesraum $F$ heisse Limesmodul über dem Limesring $R$, oder $R$-Limesmodul, wenn $F$ ein Modul über dem Ring $R$ (ein $R$-Modul) ist, so dass 1. $F$ für die zugrundeliegende additive Struktur eine (abelsche) Limesgruppe ist, and 2. die äussere Multiplikation $R \times F \rightarrow F$ stetig ist. Falls dabei $R$ ein Limeskörper $K$ ist, und $1 \cdot y=y$ für jedes $y \in F$ gilt, wobei 1 das Einselement von $K$ und der Punkt die äussere Verknüpfung in $F$ bezeichnet, so nennen wir $F$ einen Limesvektorraum über dem Limesköper $K$ oder $K$-Limesvektorraum.

Satz 15. Seien $X$ ein Limesraum, $R$ ein Limesring und $F$ ein $R$-Limesmodul. Dann ist ' ${ }^{\prime}(X, F)$, versehen mit den induzierten algebraischen Operationen, ein ' ${ }_{c}(X, R)$-Limesmodul, und zugleich ein R-Limesmodul.

Falls $F$ ein Limesvektorraum über dem Limeskörper $K$ ist, so ist $C_{c}(X, F)$ ein $K$-Limesveltorraum.

2.7. Unter einer Limesalgebra über dem Limesring $R$, oder $R$-Limesalgebra, sei ein Limesraum mit $R$-Algebra-Struktur verstanden, der für die inneren Verknüpfungen ein Limesring und für die additive innere und die äussere Verknüpfung ein $R$-Limesmodul ist. Aus den Sätzen 14 und 15 folgt

Satz 16. Seien $\mathrm{X}$ ein Limesraum, $R$ ein Limesring und $F$ eine $R$ Limesalgebra. Dann ist $C_{c}(X, F)$, versehen mit der induzierten algebraischen Struktur, eine $C_{c}(X, R)$-Limesalgebra und gleichzeitig eine R-Limesalgebra.

2.8. Im folgenden sei $R$ ein fester Limesring. Mit $\mathscr{L}$ wollen wir die Kategorie bezeichnen, deren Objekte die $R$-Limesmoduln und deren Morphismen die stetigen $R$-linearen Abbildungen zwischen solchen sind. Dann ist $\mathscr{L}$ eine Unterkategorie von $C$. Für jedes Paar $E, F$ von $R$-Limesmoduln bezeichne $\mathscr{L}(E, F)$ die Menge der Morphismen von $E$ in $F$. 
Versehen mit der von $\ell_{c}(E, F)$ induzierten $R$-Limesmodul-Struktur ist $\mathscr{L}(E, F)$ selbst wieder ein Objekt $\mathscr{L}_{c}(E, F)$ der Kategorie $\mathscr{L}$.

Sei $S$ ein beliebiger Limesraum. Wir betrachten den in 1.9 eingeführten kovarianten Funktor $C_{S}$ der Kategorie $C$ in sich. Für jedes Objekt $E$ von $\mathscr{L}$ ist nach Satz 15 der $\operatorname{Raum} \mathscr{C}_{S}(E)=\mathscr{C}_{c}(S, E)$ ein Objekt von $\mathscr{L}$, und für jeden Morphismus $f \in \mathscr{L}(E, F)$ hat man ${ }^{C}(f) \in \mathscr{L}\left(C_{S}(E), \mathscr{C}_{S}(F)\right)$. Somit stellt die Restriktion des Funktors $\mathscr{C}_{S}$ auf die Unterkategorie $\mathscr{L}$ von $\mathscr{C}$ einen kovarianten Funktor von $\mathscr{L}$ in sich dar, und die durch $C_{S}$ bestimmte Abbildung

$$
\bigodot_{S}: \mathscr{L}_{c}(E, F) \rightarrow \mathscr{L}_{c}\left({ }^{C_{S}}(E),{ }^{C_{S}}(F)\right)
$$

ist stetig und linear.

Sei $L$ ein beliebiger $R$-Limesmodul. Dieser bestimmt nach 1.9 einen kontravarianten Faktor $e^{L}$ der Kategorie ${ }^{C}$ in sich. Für jedes Objekt $X$ von $C$ ist nach Satz 15 das Bild ${ }^{C}(X)=C_{c}(X, L)$ ein $R$-Limesmodul und jeder Morphismus $f \in \mathcal{C}(X, Y)$ von $C$ wird durch $C^{L}$ in eine stetige $R$-lineare Abbildung $C^{L}(f)$ von $\Upsilon^{L}(Y)$ in $C^{L}(X)$ transformiert, wie man sofort verifiziert. Also kann $C^{L}$ auch als kontravarianter Funktor von ' $C$ in die Unterkategorie $\mathscr{L}$ betrachtet werden. Zusammenfassend können wir feststellen:

Satz 17. Seien ' $C$ die Kategorie der Limesräume und ' $\mathcal{L}$ die Kategorie der Limesmoduln über einem festen Limesring $R$.

Für jedes Objekt $S$ von $C$ ist die Restriktion des kovarianten Funktors ${ }^{\top}{ }_{S}$ auf die Unterkategorie $\mathscr{L}$ ein Funktor von $\mathscr{L}$ in sich.

Für jedes Objekt $L$ von $\mathscr{L}$ ist $C^{L}$ ein kontravarianter Funktor von ' in die Unterkategorie $\subseteq \mathcal{L}$.

2.9. Es seien $X$ und $Y$ beliebige Limesräume, $E, F$ und $G$ Limesmoduln über dem festen Limesring $R$. Nach Satz 15 sind $\bigodot_{c}\left(X, \Upsilon_{c}(Y, G)\right)$ und $\varphi_{c}(X \times Y, G) \quad R$-Limesmoduln. Der in 1.5 eingeführte natürliche Homöomorphismus $\alpha$ von $C_{c}\left(X, C_{c}(Y, G)\right)$ auf ' ${ }^{\prime}{ }_{c}(X \times Y, G)$, definiert durch $\alpha(f)=\tilde{f}, \tilde{f}(x, y)=f(x)(y)$, ist offensichtlich $R$-linear. Der Raum $\mathscr{L}_{c}\left(E, \mathscr{L}_{c}(F, G)\right)$ kann mit einem linearen Unterraum von ${ }^{\prime} C_{c}\left(E, C_{c}(F, G)\right)$ identifiziert werden. Andrerseits bezeichne $\mathscr{L}_{c}(E, F ; G)$ den linearen Unterraum von $\mathcal{C}_{c}(E \times F, G)$, bestehend aus den stetigen R-bilinearen Abbildungen. Nun ist $\mathscr{L}_{c}(E, F ; G)$ gerade das Bild von $\mathscr{L}_{c}\left(E, \mathscr{L}_{c}(F, G)\right)$ vermöge $\alpha$. Man hat also den

Satz 18. Sei $R$ ein fester Limesring. Sind $X$ und $Y$ beliebige Limesräume und ist $G$ ein R-Limesmodul, so ist die natürliche Abbildung

$$
\alpha:{ }^{\prime}\left(X, \Upsilon_{c}(Y, G)\right) \rightarrow \Upsilon_{c}(X \times Y, G)
$$


ein Isomorphismus in der Kategorie $\mathscr{L}$ der $R$-Limesmoduln. Sind $E, F$ und $G$ R-Limesmoduln, so ist die Restriktion von $\alpha$ auf den Unterraum $\mathscr{L}_{c}\left(E, \mathscr{L}_{c}(F, G)\right)$ von $\bigodot_{c}\left(E, C_{c}(F, G)\right)$ ein Isomorphismus

$$
\alpha_{0}: \mathscr{L}_{i}\left(E, \mathscr{L}_{c}(F, G)\right) \rightarrow \mathscr{L}_{c}(E, F ; G)
$$

in der Kategorie $\mathscr{L}$.

2.10. Wieder sei $R$ ein fester Limesring, und es bezeichne $\mathscr{L}$ die Kategorie der $R$-Limesmoduln. Jedes Objekt $L$ von $\mathscr{L}$ bestimmt je einen kovarianten Funktor $\mathscr{L}_{L}$ und einen kontravarianten Funktor $\mathscr{L}^{L}$ der Kategorie $\mathscr{L}$ in sich, indem man

$$
\mathscr{L}_{L}(E)=\mathscr{L}_{c}(L, E), \quad \mathscr{L}^{L}(E)=\mathscr{L}_{c}(E, L)
$$

für jeden $R$-Limesmodul $E$ setzt, und für jeden Morphismus $f \in \mathscr{L}(E, F)$ ron $\mathscr{L}$

$$
\mathscr{L}_{L}(f) \in \mathscr{L}\left(\mathscr{L}_{L}(E), \mathscr{L}_{L}(F)\right)
$$

und

$$
\mathscr{L}^{L}(f) \in \mathscr{L}\left(\mathscr{L}^{L}(F), \mathscr{L}^{L}(E)\right),
$$

definiert durch $u \rightarrow f \circ u, u \in \mathscr{L}_{c}(L, E)$, bzw. $v \longrightarrow v \circ f, v \in \mathscr{L}_{c}(F, L)$. Die Funktoren $\mathscr{L}_{L}$ bzw. $\mathscr{L}^{L}$ in der Kategorie $\mathscr{L}$ entsprechen den Funktoren $C_{S}$ bzw. ' $C$ in der Kategorie $C$. Wie für jene beweist man den

Satz 19. Sei $R$ ein fester Limesring. Für je drei R-Limesmoduln $L, E, F$ sind die durch die Funktoren $\mathscr{L}_{L}$ bzw. $\mathscr{L}^{L}$ bestimmten Abbildungen

$$
\mathscr{L}_{L}: \mathscr{L}_{c}(E, F) \rightarrow \mathscr{L}_{\mathbf{c}}\left(\mathscr{L}_{L}(E), \mathscr{L}_{L}(F)\right),
$$

$b z u$.

$$
\mathscr{L}^{L}: \mathscr{L}_{c}(E, F) \rightarrow \mathscr{L}_{c}\left(\mathscr{L}^{L}(F), \mathscr{L}^{L}(E)\right)
$$

stetig und R-linear, also Morphismen der Kategorie $\mathscr{L}$ der R-Limesräume.

2.11. Die Bezeichnungen seien wie in 2.10 Ein wichtiger Spezialfall des Funktors $\mathscr{L}^{L}$ liegt vor, wenn für $L$ der Limesring $R$ gewählt wird, wobei $R$ als Objekt von $\mathscr{L}$ betrachtet wird. Man hat dann den

Satz 20. Sei $R$ ein fester Limesring. Für jeden R-Limesmodul $E$ sei der mit der Limitierung der stetigen Konvergenz versehene Dualraum $\mathscr{L}_{c}(E, R)$ mit $E^{\prime}$ bezeichnet. Seien $E$ und $F$ zwei $R$-Limesmoduln und $u: E \rightarrow F$ eine stetige R-lincare Abbildung. Dann ist die zu $u$ duale R-lineare Abbildung $u^{\prime}: F^{\prime} \rightarrow E^{\prime}$ stetig, und die Zuordnung $u \longrightarrow u^{\prime}$ ist eine stetige R-lineare Abbildung von $\mathscr{K}_{c}(E, F)$ in $\mathscr{L}_{c}\left(F^{\prime}, E^{\prime}\right)$. 


\section{Einfache Konvergenz und getrennte Stetigkeit}

3.1. Seien $M$ eine nicht-leere Menge und $Y$ ein Limesraum. Durch Identifikation mit $Y^{M}$ wird der Menge $\mathcal{F}(M, Y)$ aller Abbildungen von $M$ in $Y$ die Produktlimitierung von $Y^{M}$ autgeprägt, nämlich die grölsste Limitierung aut $\mathcal{F}(M, Y)$, für die alle Abbildungen $u \rightarrow u(p), p \in M$, von $\mathcal{F}(M, Y)$ in $Y$ stetig sind. Wir nennen diese die Limitierung $A_{s}$ der einfachen oder punktweisen Konvergenz auf $\bar{\gamma}(M, Y)$ und setzen $\mathcal{F}_{s}(M, Y)$ statt $\left(\mathcal{F}(M, Y), A_{s}\right)$. Für einen Filter $\Theta$ auf $\mathcal{F}\left(M, Y^{r}\right)$ hat man nämlıch $\Theta \rightarrow u \in \mathcal{F}_{s}(M, Y)$ genau dann, wenn für jedes $p \in M$ gilt $\omega(\Theta \times \dot{p}) \rightarrow u(p) \in Y$. Für jede Teilmenge $H$ ron $\overline{\mathcal{F}}(M, Y)$ werde $\left(H, A_{s}\right)=H_{s}$ gesetzt. Aus der Definition von $A_{s}$ ergibt sich zunächst folgendes Stetigkeitskriterium:

Seien $T$ ein Limesraum und $H$ eine Teilmenge von $\mathcal{F}(M, Y)$. Eine Abbildung $f: T \rightarrow H_{s}$ ist genau dann stetig, wenn die Abbildung $t \rightarrow f(t)(p)$ $=\tilde{f}(t, p)$ von $T$ in $Y$ für jedes $p \in M$ stetig ist.

Nun seien $X$ und $Y$ Limesräume. Es bezeichne ' ${ }_{s}(X, Y)$ den Raum der stetigen Abbildungen von $X$ in $Y$, versehen mit der Limitierung $A_{s}$ der einfachen Konvergenz. Aus dem obigen Stetigkeitskriterium ergibt sich sofort der

Satz 21. Seien $X$ und $Y$ Limesräume und $H_{s}$ ein Unterraum ion $\mathcal{C}_{s}(X, Y)$. Für jeden Limesraum $T$ ist eine Abbildung $f: T \rightarrow H_{s}$ dann und nur dann stetig, wenn die assoziierte Abbildung $\tilde{f}: T \times X \rightarrow Y$ getrennt (d.h. argumentweise) stetig ist.

3.2. Seien wieder $X$ und $Y$ Limesräume. Die natürlichen Inklusionen

$$
i_{y}: X \rightarrow X \times Y, \quad y \in Y
$$

und

$$
j_{x}: Y \rightarrow X \times Y, \quad x \in Y
$$

definiert durch

$$
i_{y}(x)=j_{x}(y)=(x, y),
$$

sind stetig. Ist $Z$ ein weiterer Limesraum, so heisst eine Abbildung $f: X \times Y \rightarrow Z$ getrennt stetig, wenn die partiellen Abbildungen von $f$, nämlich

$$
f \circ i_{y}: \quad X \rightarrow Z \text { und } f \circ j_{x}: \quad Y \rightarrow Z,
$$

für jeden Wert von $y \in Y$, bzw. von $x \in X$, stetig sind. Um auch solche getrennt stetigen Abbildungen als Morphismen in die Kategorie $\smile$ der Limesräume einordnen zu können, führen wir eine neue Limesstruktur ein, 
und zwar auf der dem Raum $X \times Y$ zugrundeliegenden Menge, die wir als Produktmenge der Einfachheit halber auch mit $X \times Y$ bezeichnen.

Satz 22. Sind $X$ und $Y$ Limesräume, so gibt es auf der Produktmenge $X \times Y$ eine und nur eine Limitierung $\Lambda_{g}$ mit der universellen Eigenschaft:

Für jeden Limesraum $Z$ ist eine Abbildung

$$
f:\left(X \times Y, \Lambda_{g}\right) \rightarrow Z
$$

dann und nur dann stetig, wenn

$$
f: X \times Y \rightarrow Z
$$

getrennt stetig ist.

Unter allen Limitierungen auf $X \times Y$, für welche alle natürlichen Inklusionen $i_{y}(y \in Y)$, und $j_{x}(x \in X)$, stetig sind, ist $\Lambda_{g}$ die feinste.

Sei $\Lambda_{0}$ eine Limitierung auf $X \times Y$, welche der universellen Eigenschaft von Satz 22 genügt. Dann ist

$$
\text { id : } X \times Y \rightarrow\left(X \times Y, A_{0}\right)
$$

getrennt stetig, d.h. $i_{y}$ und $j_{x}$, als Abbildungen mit Werten in $\left(X \times Y, A_{0}\right)$, sind stetig. Ist $\Lambda$ eine beliebige Limitierung auf $X \times Y$, für die jedes $i_{y}$ und jedes $j_{x}$ stetig ist, so ist

$$
\text { id : }\left(X \times Y, \Lambda_{0}\right) \rightarrow(X \times Y, A)
$$

stetig, $\Lambda_{0}$ also feiner als $A$. Hieraus folgt gleichzeitig, dass $\Lambda_{z}$, falls es existiert, eindeutig bestimmt ist.

Zum Beweis der Existenz von $A_{g}$ konstruiere man für jedes feste $(x, y) \in X \times Y$ das Filterideal $\Lambda_{g}(x, y)$ auf $X \times Y$, das erzeugt wird durch alle Filter der Form $\Theta \times \dot{y}$ und $\dot{x} \times \Psi$, wo $\Phi \rightarrow x \in X$ und $\Psi \rightarrow y \in Y$. Man verifiziert sofort, dass der so konstruierten Limitierung $1_{g}$ tatsächlich die in Satz 22 geforderte universelle Eigenschaft zukommt.

Wir nennen $\Lambda_{g}$ die Limitierung der getrennten Stetigkeit auf $\mathrm{X} \times Y$ und schreiben fortan $X \times_{g} Y$ statt $\left(X \times Y, \Lambda_{g}\right)$. Das Symbol $X \times Y$ bezeichne im übrigen weiterhin das Produkt der Limesräume, wie es in 0.4 eingeführt wurde, d.h. wie bisher versehen mit der gewöhnlichen Produktlimitierung.

Bemerkungen. 1. Die Limitierung $\Lambda_{g}$ ist dann und nur dann eine Topologie auf $X \times Y$, wenn $X$ und $Y$ topologische Räume sind, wovon einer diskret.

2. Die Limitierung $\Lambda_{g}$ ist stets feiner als die Produktlimitierung, da die Inklusionen $i_{y}$ und $j_{x}$ für die letztere stetig sind. Daraus folgt, dass die Projektionen von $X \times_{g} Y$ auf $X$ und $Y$ stetig sind. 
3. Die oben durchgeführte Konstruktion lässt sich leicht auf ein Produkt von mehr als zwei Limesräumen erweitern. Sind $X, Y, Z$ Limesräume, so sei $X \times{ }_{g} Y \times{ }_{g} Z$ die Produktmenge $X \times Y \times Z$, versehen mit der Limitierung der getrennten Stetigkeit. Die Bildung ist assoziativ: Werden die Produktmengen $(X \times Y) \times Z, X \times(Y \times Z)$ und $X \times Y \times Z$ identifiziert, so hat man

$$
\left(X \times{ }_{g} Y\right) \times{ }_{g} Z=X \times{ }_{g}\left(Y \times{ }_{g} Z\right)=X \times{ }_{g} Y \times{ }_{g} Z .
$$

3.3. Wie für die gewöhnliche Produktlimitierung, hat man auch für die Limitierung der getrennten Stetigkeit den folgenden Sachverhalt:

Satz 23. Seien $X_{1}, X_{2}, Y_{1}, Y_{2}$ Limesräume und seien $f_{1}: X_{1} \rightarrow Y_{1}$, $f_{2}: X_{2} \rightarrow Y_{2}$ Abbildungen. Die Produktabbildung

$$
f_{1} \times f_{2}: X_{1} \times{ }_{g} X_{2} \rightarrow Y_{1} \times{ }_{g} Y_{2}
$$

ist dann und nur dann stetig, wenn $f_{1}$ und $f_{2}$ stetig sind.

Seien zunächst $f_{1}$ und $f_{2}$ als stetig vorausgesetzt. Für jedes feste $x_{2} \in X_{2}$ ist die erste partielle Abbildung von $f_{1} \times f_{2}$, nämlich

$$
x_{1} \longrightarrow\left(f_{1}\left(x_{1}\right), f_{2}\left(x_{2}\right)\right)
$$

stetig. weil sie sich zusammensetzt aus $f_{1}$ und der natürlichen Inklusion

$$
y_{1} \longrightarrow\left(y_{1}, f_{2}\left(x_{2}\right)\right)
$$

von $Y_{1}$ in $Y_{1} \times_{g} Y_{2}$. Dasselbe gilt, wenn $x_{1}$ und $x_{2}$ ihre Rollen tauschen. Nach Konstruktion von $X_{1} \times{ }_{g} X_{2}$ ist $f_{1} \times f_{2}$ stetig.

Wird umgekehrt $f_{1} \times f_{2}$ als stetig vorausgesetzt, so folgt, dass $f_{1}$ und $f_{2}$ stetig sind. Denn $f_{1}$ (und analog $f_{2}$ ) lässt sich wie folgt in stetige Abbildungen zerlegen:

$$
x_{1} \longrightarrow\left(x_{1}, x_{2}\right) \longrightarrow\left(f_{1}\left(x_{1}\right), f_{2}\left(x_{2}\right)\right) \longrightarrow f_{1}\left(x_{1}\right) .
$$

Dabei ist $x_{2} \in X_{2}$ beliebig.

3.4. Die Einführung der Limitierung der getrennten Stetigkeit gestattet es, dem Satz 21 eine Formulierung zu geben, die derjenigen von Satz 2 ganz analog ist.

Satz 24. Es seien $X$ und $I$ Limesräume und $H_{s}$ ein Unterraum von $\mathcal{C}_{s}(X, Y)$. Für jeden Limesraum $S$ ist eine Abbildung $f: S \rightarrow H_{s}$ dann und nur dann stetig, wenn die assoziierte Abbildung $\tilde{f}: S \times{ }_{g} X \rightarrow Y$ stetig ist.

Rein formal entsteht Satz 24 aus Satz 2 dadurch, dass $H_{c}$ durch $H_{s}$ und gleichzeitig $S \times X$ durch $S \times{ }_{g} X$ ersetzt wird. Nach Satz 3 charakterisiert die in Satz 2 ausgesprochene universelle Eigenschaft die Limitierung 
$A_{c}$ der stetigen Konvergenz, so dass sich die Eigenschaften von $\Lambda_{c}$ als Folgerungen von Satz 2 herleiten lassen, ohne dass man auf die explizite Konstruktion von $\Lambda_{c}$ zurückgreifen muss. Die formale Analogie der Sätze 2 und 24 erlaubt die Übertragung dieser Eigenschaften auf die Limitierung $A_{s}$ der einfachen Konvergenz, indem in den entsprechenden Formulierungen an geeigneter Stelle "stetig» durch "getrennt stetig» ersetzt wird.

3.5. Die folgenden drei Sätze entsprechen genau den Sätzen 4, 5 und 6 .

Satz 25. Seien $X$ und $Y$ zwei Limesräume und $K$ die Menge der konstanten Abbildungen von $X$ in $Y$. Dann ist die natürliche Bijektion if $Y \rightarrow K_{s}$ ein Homöomorphismus.

Dies bedeutet nach Satz 4, dass auf $K$ die Limitierungen $\Lambda_{c}$ und $\Lambda_{s}$ identisch sind. Da $\Lambda_{c}$ feiner als $\Lambda_{s}$ ist (vergl. Bemerkung 2 in 3.2.), bleibt zu beweisen, dass $\operatorname{id}_{K}: K_{s} \rightarrow K_{c}$ stetig ist, oder nach Satz 2, dass $\omega$ : $K_{s} \times X \rightarrow Y$ stetig ist. Diese letzte Abbildung ist aber getrennt stetig. Da ihr Wert nur vom ersten Argument abhängt, bedeutet dies bereits Stetigkeit dieser Abbildung.

Satz 26. Für drei Limesräume $X, Y$ und $Z$ definiert die Zuordnung $f \longrightarrow \tilde{f}$ einen Homöomorphismus von ${ }^{C}\left(X, C_{s}(Y, Z)\right)$ auf $\mathcal{C}_{s}\left(X \times_{g} Y, Z\right)$.

Nach Satz 24 ist die besagte Abbildung bijektiv. Dass sie ein Homöomorphismus ist, folgt wie im Beweis von Satz 5 .

Satz 27. Für drei Limesräume $X, Y, Z$ ist die durch die Komposition $(v, u) \rightarrow v \circ u$ definierte natürliche Abbildung von $\bigodot_{s}(Y, Z) \times \bigodot_{s}(X, Y)$ in ${ }_{s}(X, Z)$ getrennt stetig.

Der Beweis von Satz 6 kann wörtlich übernommen werden, wobei diesmal die auftretenden Produktmengen mit der Limitierung der getrennten Stetigkeit zu versehen sind.

Universität Zürich

Schweiz

\section{Literatur}

[1] Bastiani, Andrée: Applications différentiables et variétés différentiables de dimension infinie. - J. Analyse Math. 13, 1964, S. 1-114.

[2] Bıхz, E.: Ein Differenzierbarkeitsbegriff in limitierten Velktorräumen. - Erscheint in Comment. Math. Helv. 41.

[3] Cook, C. H., und H. R. Fischer: On equicontinuity and continuous convergence. Math. Ann. 159, 1965, S. 94-104.

[4] Fischer, H. R.: Limesräume. - Math. Ann. 137, 1959, S. 269-303.

[5] Keller, H. H.: Räume stetiger multilinearer Abbildungen als Limesräume. Math. Ann. 159, 1965, S. 259-270. 